\title{
A Network Pharmacology to Explore the Mechanism of Astragalus Membranaceus in the Treatment of Diabetic Retinopathy
}

\author{
Qi Jin $(\mathbb{D}$, Xiao-Feng Hao $\mathbb{D}$, Li-Ke Xie, Jing Xu, Mei Sun, Hang Yuan, Shi-Hui Wang, \\ Gai-Ping Wu, and Meng-Lu Miao
}

\author{
Surgical Department of Fundus Disease and Trauma, Eye Hospital, China Academy of Chinese Medical Sciences, \\ Beijing 100040, China \\ Correspondence should be addressed to Xiao-Feng Hao; fmmuhao@163.com
}

Received 19 August 2020; Revised 7 October 2020; Accepted 11 October 2020; Published 2 November 2020

Academic Editor: Subashani Maniam

Copyright $\odot 2020$ Qi Jin et al. This is an open access article distributed under the Creative Commons Attribution License, which
permits unrestricted use, distribution, and reproduction in any medium, provided the original work is properly cited.

Background. Diabetic retinopathy (DR) includes a series of typical lesions affected by retinal microvascular damage caused by diabetes mellitus (DM), which not only seriously damages the vision, affecting the life's quality of patients, but also brings a considerable burden to the family and society. Astragalus Membranaceus (AM) is a commonly used medicine in clinical therapy of eye disorders in traditional Chinese medicine (TCM). In recent years, it is also used for treating DR, but the specific mechanism is unclear. Therefore, this study explores the potential mechanism of AM in DR treatment by using network pharmacology. Methods. Based on the oral bioavailability (OB) and drug likeness (DL) of two ADME (absorption, distribution, metabolism, excretion) parameters, Traditional Chinese Medicine Systems Pharmacology Database (TCMSP), Swiss Target Prediction platform, GeneCards, and OMIM database were used to predict and screen the active compounds of AM, the core targets of AM in DR treatment. The Metascape data platform was used to perform Gene Ontology (GO) and Kyoto Encyclopedia of Genes and Genomes (KEGG) pathway enrichment analysis on the core targets. Results. 24 active compounds were obtained, such as quercetin, kaempferol, and astragaloside IV. There were 169 effective targets of AM in DR treatment, and the targets were further screened and finally, 38 core targets were obtained, such as VEGFA, AKT1, and IL-6. EGFR tyrosine kinase inhibitor resistance, AGE-RAGE signaling pathway in diabetic complications, PI3K-Akt signaling pathway, and other metabolic pathways participated in oxidative stress, cell apoptosis, angiogenesis signal transduction, inflammation, and other biological processes. Conclusion. AM treats DR through multiple compounds, multiple targets, and multiple pathways. AM may play a role in the treatment of DR by targeting VEGFA, AKT1, and IL-6 and participating in oxidative stress, angiogenesis, and inflammation.

\section{Introduction}

Diabetes mellitus (DM) is a metabolic disorder caused by genetic and environment factors, and it has become a critical health problem worldwide due to its high prevalence and related disability and mortality [1]. In a recent cross-sectional study, it was observed that the prevalence of diabetes had increased slightly among adults living in China from 2007 to 2017 [2]. Paralleling with the increasing pandemic of $\mathrm{DM}$, diabetic retinopathy (DR) is one of the complications of diabetes. The prevalence of DR is also growing, and an epidemiological study from urban Chinese indicated that the occurrence of DR was $8.1 \%$ for patients with DM [3]. DR destroyed the normal function of the retina and was a threat for sight in adults aged 20-74 years [4], which seriously affects the quality of patients' life [5]. Moreover, DR needs repeated treatment, which brings heavy financial burden to the family and society [6]. Therefore, early diagnosis and early treatment are particularly important. Clinically, most conventional treatments such as maintaining blood glucose and lipids and keeping a healthy lifestyle are adopted. However, there are certain limitations, so how to effectively prevent and treat DR has become a current research hotspot [7].

Recently, traditional Chinese medicine (TCM) is gradually attracting public's attention owing to its clinical efficacy. Compared with western medicine, TCM is an important alternative strategy because of syndrome 
differentiation and holistic concept for treating DM [8]. Also, there are various treatment principles for $D R$, including boosting qi and nourishing yin, fortifying the spleen and removing dampness, invigorating blood, and unblocking the collaterals [9]. Through treatment according to pattern differentiation, herbal medicines have certain advantages in preventing and treating DR [10].

Astragalus Membranaceus (AM) is named "Huangqi" of TCM. AM was originally described in the Sheng Nong's Herbal Classic which is the Classic of Herbal Medicine in ancient TCM practice, which is the dry root of astragalus mongolicus or membranous astragalus [11]. It is one of the popular health-promoting herbs that have been used to strengthen immunity of people for more than 2000 years. Furthermore, it contains numerous constituents, such as saponins, flavonoids, and polysaccharides, which have wide biological activities, for example, anti-inflammatory, antithrombotic, antioxidant, and antidiabetic activities. Chinese herbs have been used since centuries for the treatment and prevention of various disorders. There were studies that AM was found upregulating insulin-signaling pathways by improving the activity of casein kinase, regulating lipid metabolism, and enhancing insulin resistance to treat T2DM [12]. Owing to properties that they may alleviate several hyperglycaemia-induced pathological occurrences in the retina, AM can potentially be used for the treatment and prevention of DR [13]. There were numerous studies which reported that AM could downregulate the signaling pathway of p38 MAPK, simultaneously inhibit the mediated pathway of $\mathrm{NF}-\kappa \mathrm{B}$, and reduce the production of proinflammatory cytokines, such as IL- $1 \beta$ and TNF- $\alpha$, which were induced by AGE. Moreover, it can inhibit the formation of retinal angiogenesis by downregulating the level of VEGF in Muller cells induced by hyperglycemia [14].

However, the specific mechanism of action is not clear. In recent years, a new trend emerged by using network pharmacology to understand TCM. From the perspective of systems biology, network pharmacology explores the relationship between drugs-components-targets-diseases, predicts the potential mechanism of drugs to treat diseases, and provides a theoretical basis for effectiveness of TCM to treat the disease [15]. Therefore, this study used the method of network pharmacology, collecting data and information from multiple data platforms and exploring the potential mechanism of AM in DR treatment, to provide a theoretical basis for the prevention and treatment of DR by AM. The overall flow chart of the study is shown in Figure 1.

\section{Materials and Methods}

2.1. Screening of Potential Compounds and Targets of the Herb. "Huangqi" (AM) was retrieved in TCMSP and screened with $\mathrm{OB} \geq 30 \%$ and $\mathrm{DL} \geq 0.18$. The chemical compounds of AM were obtained, and the compounds of AM from the literature reports were added. All the components of AM were combined. The PubChem platform (https://pubchem.ncbi.nlm.nih.gov/, 2020.6.04) platform was used, and the InCHIKey number or CAS number of the effective components was entered in order to obtain the $2 \mathrm{D}$ structural formula of the components. And then, the $2 \mathrm{D}$ structural formula was imported into the Swiss Target Prediction platform (http://www.swisstargetprediction.ch/), 2020.6.04 [16]. The species was selected to be "Homo sapiens," and the potential targets of the compounds with the condition of "probability $\geq 0$ " were predicted. Finally, targets obtained from both databases were combined and deduplicated, and the targets of AM were obtained.

TCMSP: The Traditional Chinese Medicine Systems Pharmacology Database and Analysis Platform (TCMSP) (https://tcmspw.com/tcmsp.php) is a database that is based on the framework of systems pharmacology for herbal medicine, which can predict the compound and target of herbal medicines by setting ADME (absorption, distribution, metabolism, excretion) parameters [17].

ADME parameters: Most herbal medicines are mainly taken orally and undergo metabolic processes, such as metabolism, absorption, distribution, and excretion (ADME) in the human body, including OB and DL.

Oral bioavailability (OB): $\mathrm{OB}$ refers to the speed and degree of absorption of the active compounds or active groups of the drug in the systemic circulation, and is a key parameter for evaluating whether the drug can be developed. Generally, molecules with $\mathrm{OB} \geq 30 \%$ have good oral availability [18].

Drug likeness (DL): the DL of all herbal medicines is acquired by calculation of Tanimoto coefficient. The Tanimoto coefficient is defined as follows [19]:

$$
f(A, B)=\frac{A \cdot B}{|A|^{2}+|B|^{2}-A \cdot B} .
$$

Here, $A$ is the molecular descriptor index of the compounds of herbal medicines to be tested in the TCMSP database and $B$ is the average drug likeness index described by all 6511 small drug molecules in the DrugBank database. The descriptors of all molecules are calculated by the Dragon software (http://www.talete.mi.it/index.htm). Generally, molecules with $\mathrm{DL} \geq 0.18$ have good drug likeness.

PubChem: PubChem is the world's largest collection platform of chemical information and search information of compounds, such as chemical and physical properties, biological activities, safety, and toxicity information and more and information can be obtained by importing name, molecular formula, structure, and other identifiers of compounds [20].

Swiss Target Prediction: Swiss Target Prediction is a type of online analysis software for predicting targets of small molecules, which can import SMILES and structure of the molecule to predict the targets of small molecule compounds [21].

2.2. Screening of Potential Targets of the Disease. The keyword "diabetic retinopathy" was searched in the GeneCards Database (https://www.genecards.org/, 2020.6.05) [22], and the related targets of DR were obtained. In the OMIM database (https://omim.org/,2020.6.05) [23], Gene 


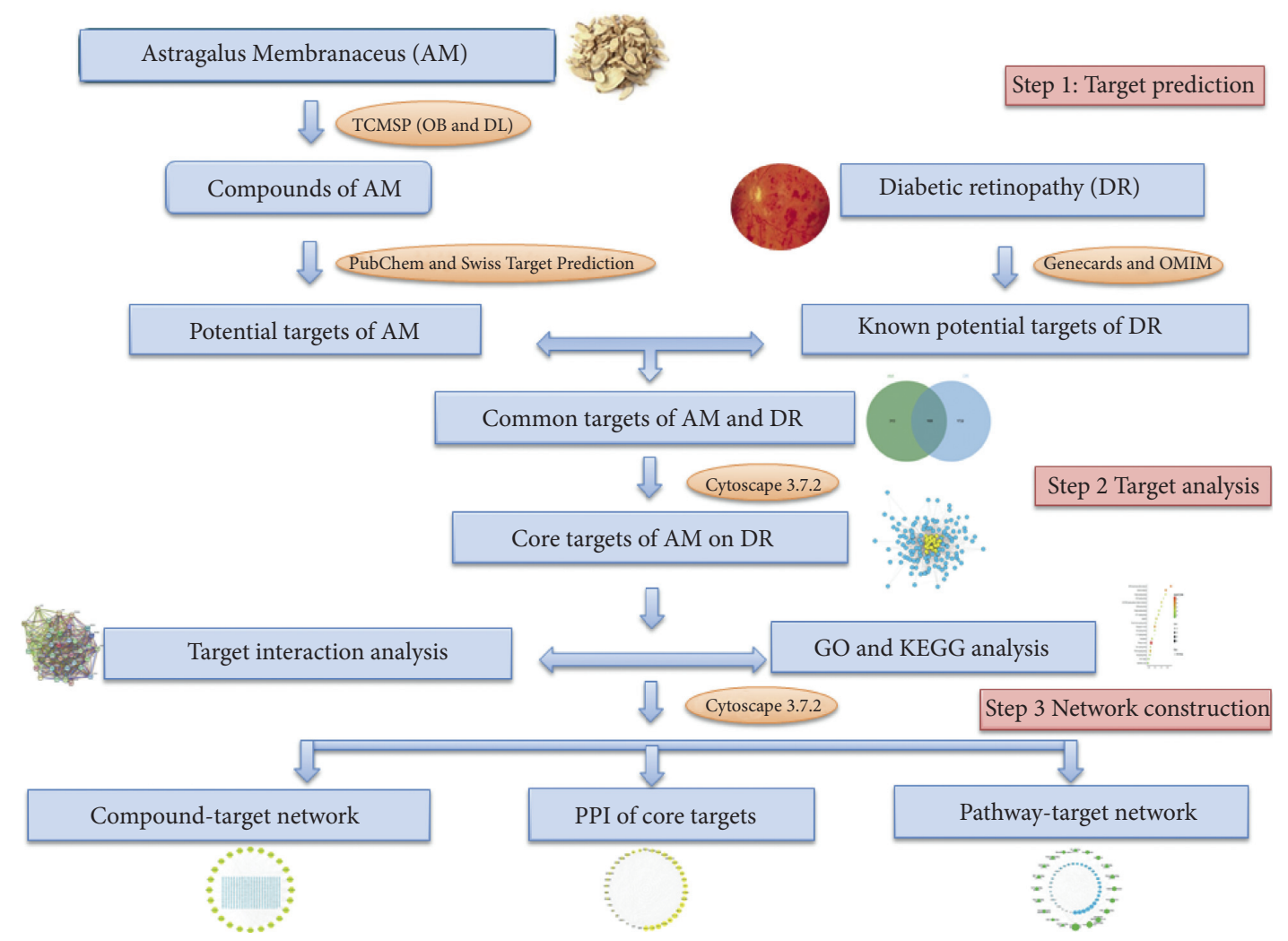

Figure 1: Flow chart of the study. DR: diabetic retinopathy; AM: Astragalus Membranaceus; TCMSP: Traditional Chinese Medicine Systems Pharmacology Database; GO: Gene Ontology; KEGG: Kyoto Encyclopedia of Genes and Genomes; PPI: protein-protein interaction.

Map was selected in "Advanced Search" and then the keyword "diabetic retinopathy" was entered, and the related targets of DR were obtained. The targets obtained from the two databases were integrated and duplicated, and the targets of disease were obtained.

Online Mendelian Inheritance in Man (OMIM) and GeneCards are two different databases that provide comprehensive information of all annotated and predicted human genes.

\subsection{Screening of Core Targets of AM in DR Treatment.} The jvenn online platform (http://jvenn.toulouse.inra.fr/ app/example.html) [24] was used for core targets. The targets of the drug and disease were, respectively, imported, and the intersection targets between drug and disease were obtained. Then, the String platform (https://string-db.org/) was used and protein-protein interaction (PPI) was built. In the platform, "Multiple proteins" was selected and the organism was selected as "Homo sapiens." The intersection targets of AM and DR were then imported, the medium confidence of minimum required interaction score was set as 0.4 , and the remaining parameters were maintained at the default settings [25]. The file was exported in "TSV format," and then, Cytoscape 3.7.2 was used to analyze the topological properties of the PPI network. The degree values were calculated, and the core targets of AM in DR treatment with degree $\geq 2 \times$ median were selected.
STRING: it is a search tool for the retrieval of interacting genes/proteins, which is applied for predicting the PPI network and detecting the possible relationships.

Cytoscape: it is a type of open-source software used to analyze and visualize biological networks [26].

2.4. GO and KEGG Pathway Enrichment Analysis. The core targets of AM in DR treatment were imported into the Metascape platform, and in this, species was selected as " $\mathrm{H}$ sapiens," "Custom analysis" was selected, and parameters of $P$ value $<0.01$, a minimum count of 3 , and an enrichment factor $>1.5$ (the enrichment factor is the ratio between the observed counts and the counts expected by chance) were set. GO and KEGG pathway enrichment analyses on core targets were performed.

Metascape: it is a gene function annotation analysis tool, to realize the recognition of gene or protein function through the bioinformatics analysis of genes and proteins [27].

Gene Ontology (GO): it is a bioinformatics tool used for annotating genes, gene products, and sequences to analyze their biological processes. GO includes 3 aspects: molecular function (MF); biological process (BP); cellular component (CC).

KEGG: it is a database resource comprising information on the biological systems of genome sequences and other high-throughput data [28]. 


\subsection{Construction of Networks}

2.5.1. Compound-Target Network. Cytoscape 3.7.2 was used to construct the component-target network (C-T network).

2.5.2. PPI of Core Targets. String online platform was used to build PPI of core targets, and then, PPI was visualized by Cytoscape 3.7.2.

2.5.3. Target-Pathway Network. The top 20 KEGG pathways were screened, and Cytoscape 3.7.2 was used to construct the target-pathway network (T-P network).

\section{Results}

3.1. Active Compounds and Targets of the Drug. On the TCMSP platform, the ADME parameters of $\mathrm{OB} \geq 30 \%$ and $\mathrm{DL} \geq 0.18$ were selected 20 active compounds were obtained, and then, according to the relevant literature [29], additional 4 active compounds were obtained and merged with the former, and finally, a total of 24 compounds of AM were identified (Table 1).

2D structural formulas of 24 compounds were obtained from PubChem, and then, compounds were imported into the Swiss Target Prediction platform to obtain the target information of 24 compounds. Targets were merged and deduplicated, and a total of 482 targets of AM were obtained. The component-target network (C-T network) was constructed (Figure 2) by using Cytoscape 3.7.2, and there were 506 nodes and 1310 edges. The function of "Network Analyzer" in Cytoscape 3.7.2 was used to perform topological analysis on the components (Table 2), and the main compounds were jaranol, isoflavanone, kaempferol, and quercetin.

3.2. Core Targets of AM on DR. There were 217 targets of $D R$ in the OMIM database, while there were 1753 targets of DR in the GeneCards database. The targets from the two databases were merged, the duplicates were removed, and finally, 1901 targets of DR were obtained. 482 targets of AM and 1901 targets of DR were imported into Venny2.1.0 online platform, and there were 169 common targets (Figure 3). 169 targets were imported into STRING online platform, and a file in "TSV" format was exported. Then, it was imported into Cytoscape 3.7.2, the function of "network analyzer" was used, and the median of degree was set as 18 . 38 core targets of AM in DR treatment with degree $\geq 36$ $(2 \times 18)$ were screened (Figure 4$)$.

3.3. PPI of Core Targets. 38 core targets were imported into the STRING platform, the PPI of the core targets were obtained (Figure 5), and finally, there were 38 nodes and 555 edges. The average node degree was 29.2. The specific information of targets is shown in Table 3.

3.4. Enrichment Analysis of 38 Targets. There were 38 targets of AM on DR; the biological function of the 38 targets was elucidated. GO analysis and KEGG pathway analysis on 38 targets were performed. Based on GO results, there were 79 processes of molecular functions, such as protein kinase activity, protein serine/threonine kinase activity, and kinase binding; there were 418 biological processes, such as transmembrane receptor protein tyrosine kinase signaling pathway, positive regulation of cell migration, and positive regulation of cell motility; there were 42 cellular components, such as membrane raft, membrane microdomain, and membrane region. In addition, there were 123 pathways for results of KEGG.

The top 20 results of KEGG were listed and the scatter plot was drawn. The larger the dot, the more the count, the redder the color, and the smaller the $P$ value (Figure 6), and EGFR tyrosine kinase inhibitor resistance, AGERAGE signaling pathway in diabetic complications, PI3KAKT signaling pathway and IL-17 signaling pathway may be involved in the treatment of DR by AM. The top 20 pathways were screened and the target-pathway network (T-P network) was constructed (Figure 7). There were 55 nodes and 331 edges, and the topology analysis was further analyzed. The main pathways included EGFR tyrosine kinase inhibitor resistance, AGE-RAGE signaling pathway in diabetic complications, and PI3K-Akt signaling pathway (Table 4 ).

\section{Discussion}

$\mathrm{DR}$ is a common and severe complication of DM, which is the main cause of blindness in adults. In the early stage, the high-glucose environment influences the retinal microvascular damage, which is characterized by loss of retinal cells, thickening of the basement membrane of capillaries, expansion and deformation of capillaries, and destruction of the blood-retinal barrier. Moreover, the progression is characterized by the occlusion of capillaries and the formation of pathological neovascularization, retinal hemorrhage, etc, which cause irreversible loss of vision [30]. Experts and scholars have been devoted to the research of effective prevention and treatment of DR, but there are still many unsolved mysteries. In recent years, studies reported that the treatment of this disease by TCM had been increasing year by year and the treatment efficiency had gradually improved. AM is a class of herbs used for boosting qi and invigorating blood, but its precise mechanism of AM on DR is not yet clear. Therefore, it is necessary to explore the mechanism of AM in DR treatment by using functions of screening compounds, predicting targets, and analyzing pathways of drugs in network pharmacology.

In this study, we firstly identified the active compounds of AM and combined literature reviews. 24 compounds were identified, and the specific information of compounds is shown in Table 1. Some compounds, such as quercetin, kaempferol, and astragaloside IV were reported to have played an important role in DR. Quercetin could reduce the expression of MMP-9 and VEGF in rats with diabetic retinopathy [31] and inhibit the generation of angiogenesis both in vitro and ex vivo [32]. Quercetin can reduce the overproduction of MCP, IL-6, and ROS and downregulate the expression of P53, 
TABLE 1: Information of compounds from AM.

\begin{tabular}{|c|c|c|c|c|c|}
\hline Number & Mol ID & Molecule name & $\begin{array}{c}\text { Molecule } \\
\text { weight }\end{array}$ & $\begin{array}{l}\text { OB } \\
(\%) \\
\end{array}$ & DL \\
\hline 1 & MOL000211 & Mairin & 456.78 & 55.38 & 0.78 \\
\hline 2 & MOL000239 & Jaranol & 314.31 & 50.83 & 0.29 \\
\hline 3 & MOL000296 & Hederagenin & 414.79 & 36.91 & 0.75 \\
\hline 4 & MOL000033 & $\begin{array}{l}\text { (3S,8S,9S,10R,13R,14S,17R)-10,13-dimethyl-17-[(2R,5S)-5-propan-2-yloctan-2- } \\
\text { yl]-2,3,4,7,8,9,11,12,14,15,16,17-dodecahydro-1H-cyclopenta (a) phenanthren-3-ol }\end{array}$ & 428.82 & 36.23 & 0.78 \\
\hline 5 & MOL000354 & Isorhamnetin & 316.28 & 49.6 & 0.31 \\
\hline 6 & MOL000371 & 3,9-di-O-Methylnissolin & 314.36 & 53.74 & 0.48 \\
\hline 7 & MOL000374 & $5^{\prime}$-Hydroxyiso-muronulatol-2' $5^{\prime}$-di-O-glucoside & 642.67 & 41.72 & 0.69 \\
\hline 8 & MOL000378 & 7-O-Methylisomucronulatol & 316.38 & 74.69 & 0.3 \\
\hline 9 & MOL000379 & 9,10-Dimethoxypterocarpan-3-O- $\beta$-D-glucoside & 462.49 & 36.74 & 0.92 \\
\hline 10 & MOL000380 & $\begin{array}{l}\text { (6aR,11aR)-9,10-Dimethoxy-6a,11a-dihydro-6H-benzofurano }(3,2-c) \text { chromen-3- } \\
\text { ol }\end{array}$ & 300.33 & 64.26 & 0.42 \\
\hline 11 & MOL000387 & Bifendate & 418.38 & 31.1 & 0.67 \\
\hline 12 & MOL000392 & Formononetin & 268.28 & 69.67 & 0.21 \\
\hline 13 & MOL000398 & Isoflavanone & 316.33 & 109.99 & 0.3 \\
\hline 14 & MOL000417 & Calycosin & 284.28 & 47.75 & 0.24 \\
\hline 15 & MOL000422 & Kaempferol & 286.25 & 41.88 & 0.24 \\
\hline 16 & MOL000433 & FA & 441.45 & 68.96 & 0.71 \\
\hline 17 & MOL000438 & (3R)-3-(2-Hydroxy-3,4-dimethoxyphenyl)chroman-7-ol & 302.35 & 67.67 & 0.26 \\
\hline 18 & MOL000439 & Isomucronulatol-7, $2^{\prime}$-di-O-glucosiole & 626.67 & 49.28 & 0.62 \\
\hline 19 & MOL000442 & 1,7-Dihydroxy-3,9-dimethoxy pterocarpene & 314.31 & 39.05 & 0.48 \\
\hline 20 & MOL000098 & Quercetin & 302.25 & 46.43 & 0.28 \\
\hline 21 & MOL000401 & Astragaloside I & 869.17 & 46.79 & 0.11 \\
\hline 22 & MOL000403 & Astragaloside II & 827.13 & 46.06 & 0.13 \\
\hline 23 & MOL000405 & Astragaloside III & 785.09 & 31.83 & 0.1 \\
\hline 24 & MOL000407 & Astragaloside IV & 785.09 & 22.5 & 0.15 \\
\hline
\end{tabular}

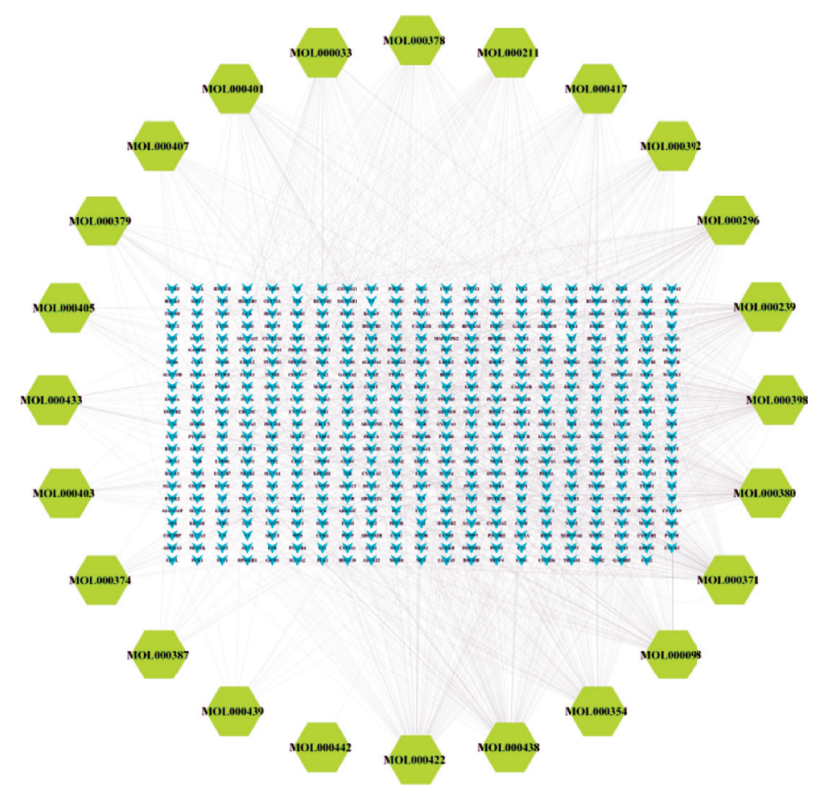

FIGURE 2: C-T network. The layout of the outer ring and the inner rectangular were constructed according to different nodes, and the green nodes represent the compound while blue represents the target; the edges among the nodes represent the relationship between the compound and the targets. The more the edges of nodes, the more important the nodes.

Bax, and Caspase-3 by promoting PTEN/AKT pathway and inhibiting NF- $\kappa \mathrm{B}$ pathway, which could protect ARPE-19 cell damage induced by high glucose [33]. Kaempferol could protect against oxidative stress leading to damage of human RPE cells through its antioxidant activity and antiapoptosis function [34]. Moreover, it could suppress angiogenesis of human retinal endothelial cells (HRECs) via targeting VEGF and PGF to inhibit the activation of Src-Akt1-Erk1/2 signaling pathway under glucose conditions [35]. Astragaloside IV could prevent 
TABLE 2: Key compounds of AM.

\begin{tabular}{|c|c|c|c|c|}
\hline Mol ID & Name & $\mathrm{DC}$ & $\mathrm{CC}$ & $\mathrm{BC}$ \\
\hline MOL000422 & Kaempferol & 100 & 0.390867 & 0.078562 \\
\hline MOL000354 & Isorhamnetin & 100 & 0.390867 & 0.070723 \\
\hline MOL000098 & Quercetin & 100 & 0.390867 & 0.070715 \\
\hline MOL000239 & Jaranol & 100 & 0.390263 & 0.09292 \\
\hline MOL000438 & (3R)-3-(2-Hydroxy-3,4-dimethoxyphenyl)chroman-7-ol & 100 & 0.38966 & 0.14801 \\
\hline MOL000398 & Isoflavanone & 100 & 0.38906 & 0.212952 \\
\hline MOL000371 & 3,9-di-O-Methylnissolin & 100 & 0.38727 & 0.212242 \\
\hline MOL000380 & (6aR,11aR)-9,10-Dimethoxy-6a,11a-dihydro-6H-benzofurano (3,2-c)chromen-3-ol & 100 & 0.386677 & 0.13606 \\
\hline
\end{tabular}

$\mathrm{DC}=$ degree centrality; $\mathrm{CC}=$ closeness centrality; $\mathrm{BC}=$ betweenness centrality.

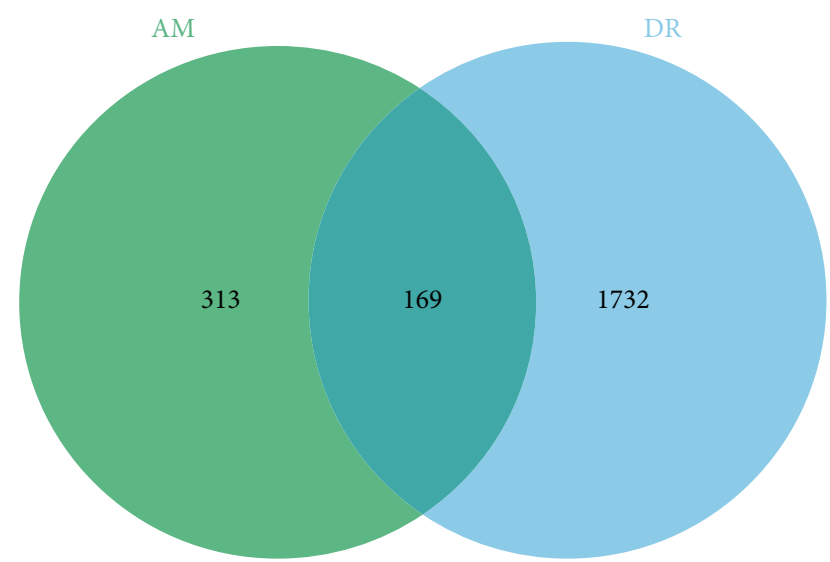

FIGURE 3: Common targets of AM and DR. There were 482 targets of AM (left), while 1901 targets of DR (right), and 169 common targets between AM and DR (middle).

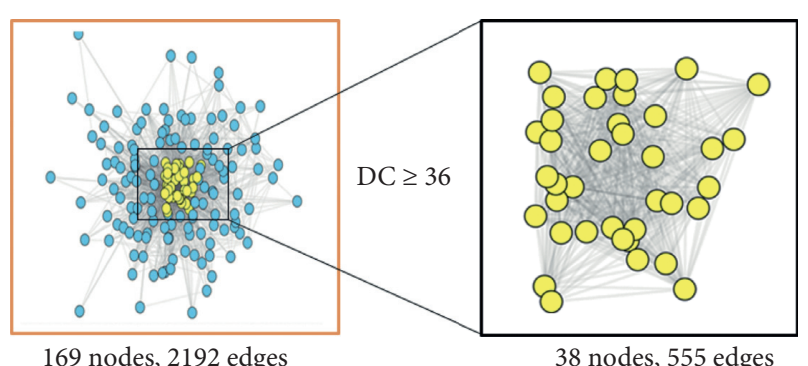

FIgURE 4: The process of screening core targets. From 169 common targets (left), selection of 38 core targets with degree $\geq 36$ (right).

the activation of ERK1/2 phosphorylation and NF- $\kappa \mathrm{B}$ and further relieve the RGCs dysfunction in $\mathrm{ab} / \mathrm{db}$ mice with DR [36], and it has potential protective effects on retinal capillary endothelial cells (RCECs) incubated with high glucose conditions [37].

We predicated 169 common targets between AM and $\mathrm{DR}$, and on further screening, 38 core targets for AM in DR treatment were obtained. The targets were mainly enriched for oxidative stress, cell proliferation, apoptosis, inflammation, etc. When it comes to core targets, Akt1, VEGFA, IL-6, Caspase-3, and STAT3 played an important role in the process of AM in DR treatment. The detailed information is given in Table 2. VEGFA could be an activator of the

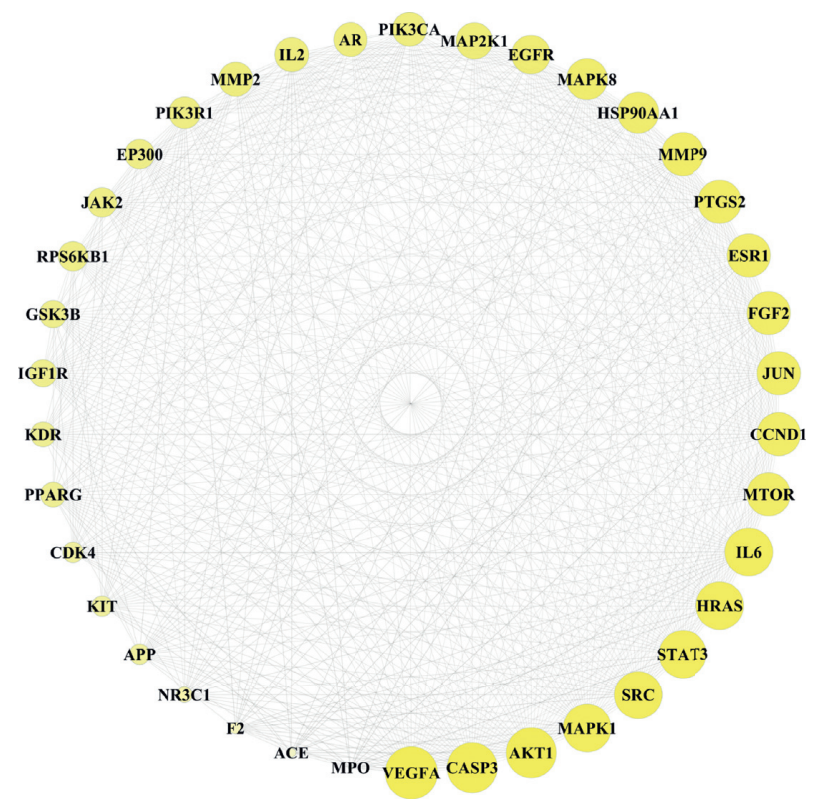

FIGURE 5: PPI of core targets. The layout of the outer ring went in an anticlockwise direction according to the area and color of the nodes, andthe yellow nodes represent the core targets of AM on DR; the size and transparency of the nodes were divided according to the degree value from large to small. The inner edges represent the relationship between the core targets. The more the edges of the nodes were, the larger the area of the nodes, the darker the color, and the more important the targets.

stimulation of angiogenesis, and particularly, it was reported to be strongly upregulated in PDR [38]. Numerous studies had reported that AKT signaling pathway could be involved in the occurrence and development of DR and the activation of AKT can maintain the steady state of endothelial function of retinal capillaries [39]. Caspase-3 could participate in the pathological process of DR by regulating apoptosis, and the abnormally high expression level of Caspase- 3 may be correlated with the severity of DR [40]. The inhibition of STAT13 can alleviate retinal inflammation and reduce retinal cell death in rats of DR, while IL- 6 could also be a proinflammatory factor, and and by inhibiting the activation of IL-6/STAT3 signaling pathway and then affecting the oxidation and antioxidation process of the retina, the purpose of protecting the retina of diabetic rats can be achieved [41]. 
TABLE 3: Specific information for core targets.

\begin{tabular}{|c|c|c|c|c|}
\hline Number & Uniprot ID & Gene name & Protein name & Counts \\
\hline 1 & P31749 & AKT1 & RAC-alpha serine/threonine-protein kinase & 36 \\
\hline 2 & P15692 & VEGFA & Vascular endothelial growth factor A & 37 \\
\hline 3 & P05231 & IL6 & Interleukin-6 & 35 \\
\hline 4 & P42574 & CASP3 & Caspase-3 & 36 \\
\hline 5 & P12931 & SRC & Proto-oncogene tyrosine-protein kinase Src & 35 \\
\hline 6 & P00533 & EGFR & Epidermal growth factor receptor & 31 \\
\hline 7 & P28482 & MAPK1 & Mitogen-activated protein kinase 1 & 35 \\
\hline 8 & P40763 & STAT3 & Signal transducer and activator of transcription 3 & 35 \\
\hline 9 & P05412 & JUN & Transcription factor AP-1 & 33 \\
\hline 10 & $\mathrm{P} 01112$ & HRAS & GTPase HRas & 35 \\
\hline 11 & P07900 & HSP90AA1 & Heat shock protein HSP 90 -alpha & 32 \\
\hline 12 & P45983 & MAPK8 & Mitogen-activated protein kinase 8 & 32 \\
\hline 13 & P42345 & MTOR & Serine/threonine-protein kinase mTOR & 33 \\
\hline 14 & P24385 & CCND1 & G1/S-specific cyclin-D1 & 33 \\
\hline 15 & P03372 & ESR1 & Estrogen receptor & 33 \\
\hline 16 & P09038 & FGF2 & Fibroblast growth factor 2 & 33 \\
\hline 17 & $\mathrm{P} 14780$ & MMP9 & Matrix metalloproteinase- 9 & 33 \\
\hline 18 & P35354 & PTGS2 & Prostaglandin $\mathrm{G} / \mathrm{H}$ synthase 2 & 33 \\
\hline 19 & P37231 & PPARG & Peroxisome proliferator-activated receptor gamma & 25 \\
\hline 20 & $\mathrm{P} 42336$ & PIK3CA & Phosphatidylinositol 4,5-bisphosphate 3-kinase catalytic subunit alpha isoform & 29 \\
\hline 21 & P10275 & AR & Androgen receptor & 29 \\
\hline 22 & P08253 & MMP2 & $72 \mathrm{kDa}$ type IV collagenase & 29 \\
\hline 23 & P35968 & KDR & Vascular endothelial growth factor receptor 2 & 25 \\
\hline 24 & Q09472 & $\mathrm{EP} 300$ & Histone acetyltransferase p300 & 27 \\
\hline 25 & P27986 & PIK3R1 & Phosphatidylinositol 3-kinase regulatory subunit alpha & 28 \\
\hline 26 & P60568 & IL2 & Interleukin-2 & 29 \\
\hline 27 & P12821 & ACE & Angiotensin-converting enzyme & 18 \\
\hline 28 & O60674 & JAK2 & Tyrosine-protein kinase JAK2 & 27 \\
\hline 29 & P05067 & APP & Amyloid-beta precursor protein & 23 \\
\hline 30 & Q02750 & MAP2K1 & Dual specificity mitogen-activated protein kinase kinase 1 & 30 \\
\hline 31 & P08069 & IGF1R & Insulin-like growth factor 1 receptor & 26 \\
\hline 32 & P05164 & MPO & Myeloperoxidase & 16 \\
\hline 33 & P23443 & RPS6KB1 & Ribosomal protein S6 kinase beta-1 & 27 \\
\hline 34 & P04150 & NR3C1 & Glucocorticoid receptor & 21 \\
\hline 35 & P10721 & KIT & Mast/stem cell growth factor receptor kit & 23 \\
\hline 36 & P49841 & GSK3B & Glycogen synthase kinase- 3 beta & 26 \\
\hline 37 & P11802 & $\mathrm{CDK} 4$ & Cyclin-dependent kinase 4 & 23 \\
\hline 38 & $\mathrm{P} 00734$ & $\mathrm{~F} 2$ & Prothrombin & 19 \\
\hline
\end{tabular}

Subsequently, we compared the results of GO and KEGG enrichment analyses for 38 key targets, and the results indicated that regulation of various kinase activities, response to insulin, and regulation of vascular endothelial cells played a main role in the process of AM in DR treatment. Furthermore, in delaying the progression of diabetic retinopathy, oxidative stress and inflammation could also play an important role [42]. The action pathway mainly includes EGFR tyrosine kinase inhibitor resistance, AGE-RAGE signaling pathway in diabetic complications, and PI3K-Akt signaling pathways. Previous studies had indicated that renal epidermal growth factor receptors (EGFRs) were activated in models of diabetic nephropathy (DN), and inhibition of EGFR activity protected against progressive DN in T1 DM and T2 DM [43]. So the EGFR tyrosine kinase inhibitor resistance may delay the progression of diabetes and improve complications such as diabetic retinopathy. In the condition of high glucose, nonenzymatic glycosylation of proteins and lipids can be induced, advanced glycation endproducts (AGEs) can be generated, and through the activation of AGE receptor (RAGE), oxidative stress and inflammation can be induced [44], which could damage the retina. The details of AM on the AGEs-RAGE pathway are shown in Figure 8, and it was cited from the KEGG database (https://www.kegg.jp/).

In addition, there were several pathways, such as pathway in cancer, proteoglycans in cancer, and hepatitis bare, indicating AM might exhibit potential therapeutic effects on these diseases, while demonstrating that the progression of DR is influenced by other diseases. There were multiple targets, multiple pathways, and multiple processes involved in treatment of DR through AM. In addition, there were still some deficiencies in this study. The network pharmacology method was used to predict the 


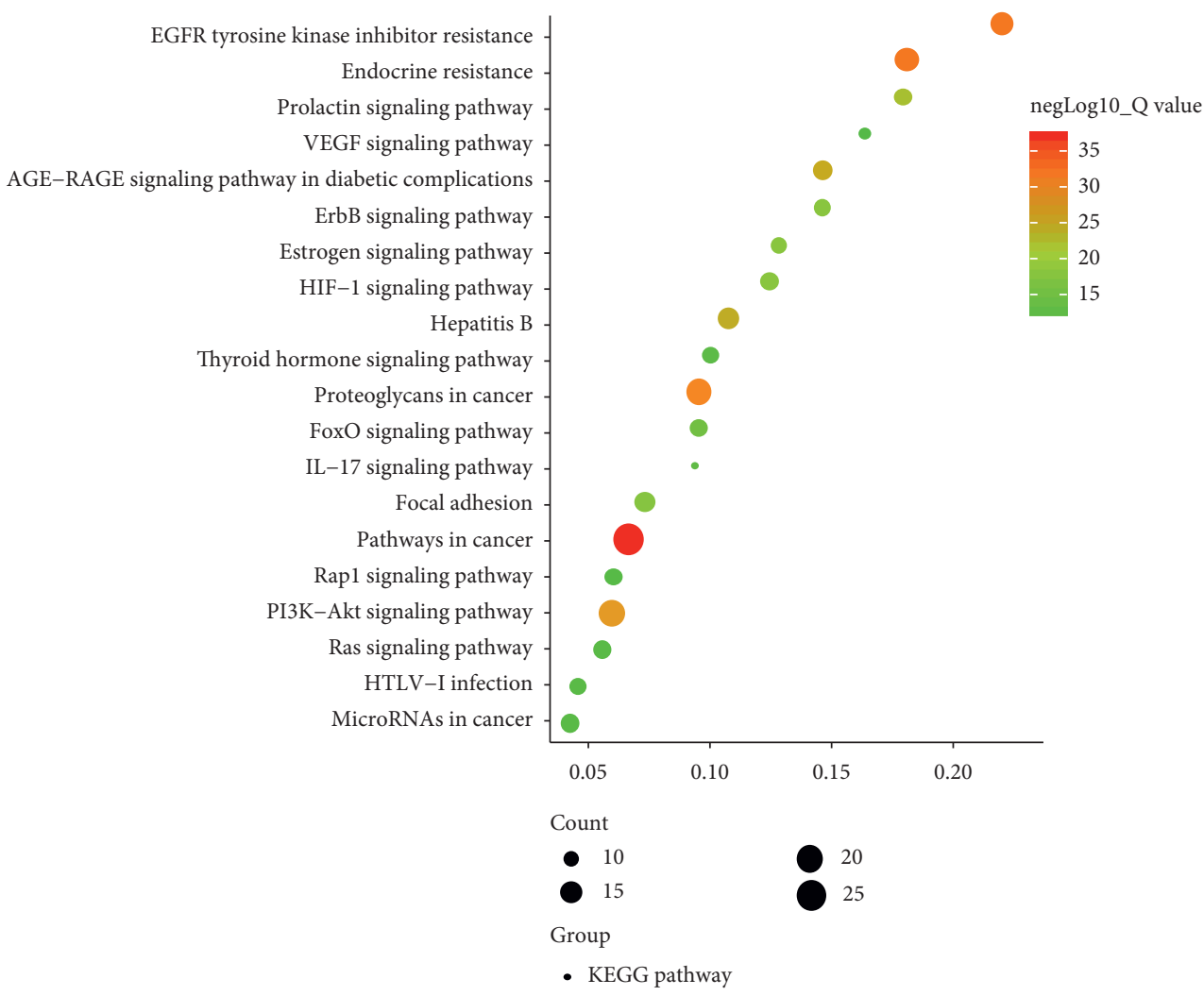

Figure 6: The top 20 results of KEGG pathway. The larger the area, the more the count, the redder the color, and the smaller the $P$ value.

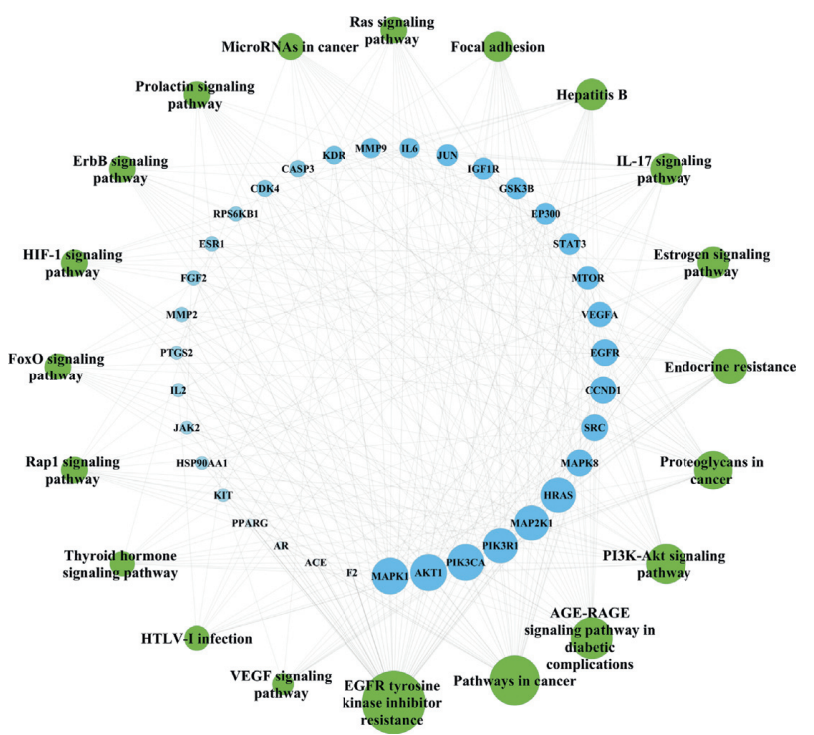

Figure 7: T-P network. The layout of the outer and inner ring went in an anticlockwise direction according to the area and color of the nodes; the green nodes represent the pathways while the blue nodes represent targets. The size and transparency of the nodes were divided according to the degree value from large to small. The inner edges represented the relationship between the pathways and the targets. The more edges, the larger the area of the nodes, the darker of the color, and the more important the nodes. 
TABLE 4: The main pathway of KEGG.

\begin{tabular}{|c|c|c|c|c|}
\hline Term & Name & $\mathrm{BC}$ & $\mathrm{CC}$ & $\mathrm{DC}$ \\
\hline hsa01521 & EGFR tyrosine kinase inhibitor resistance & 0.23113 & 0.739726 & 35 \\
\hline hsa05200 & Pathways in cancer & 0.094192 & 0.606742 & 27 \\
\hline hsa04933 & AGE-RAGE signaling pathway in diabetic complications & 0.044391 & 0.545455 & 22 \\
\hline hsa04151 & PI3K-Akt signaling pathway & 0.049164 & 0.534653 & 21 \\
\hline hsa05205 & Proteoglycans in cancer & 0.036825 & 0.524272 & 20 \\
\hline
\end{tabular}

$\mathrm{DC}=$ degree centrality; $\mathrm{CC}=$ closeness centrality; $\mathrm{BC}=$ betweenness centrality.

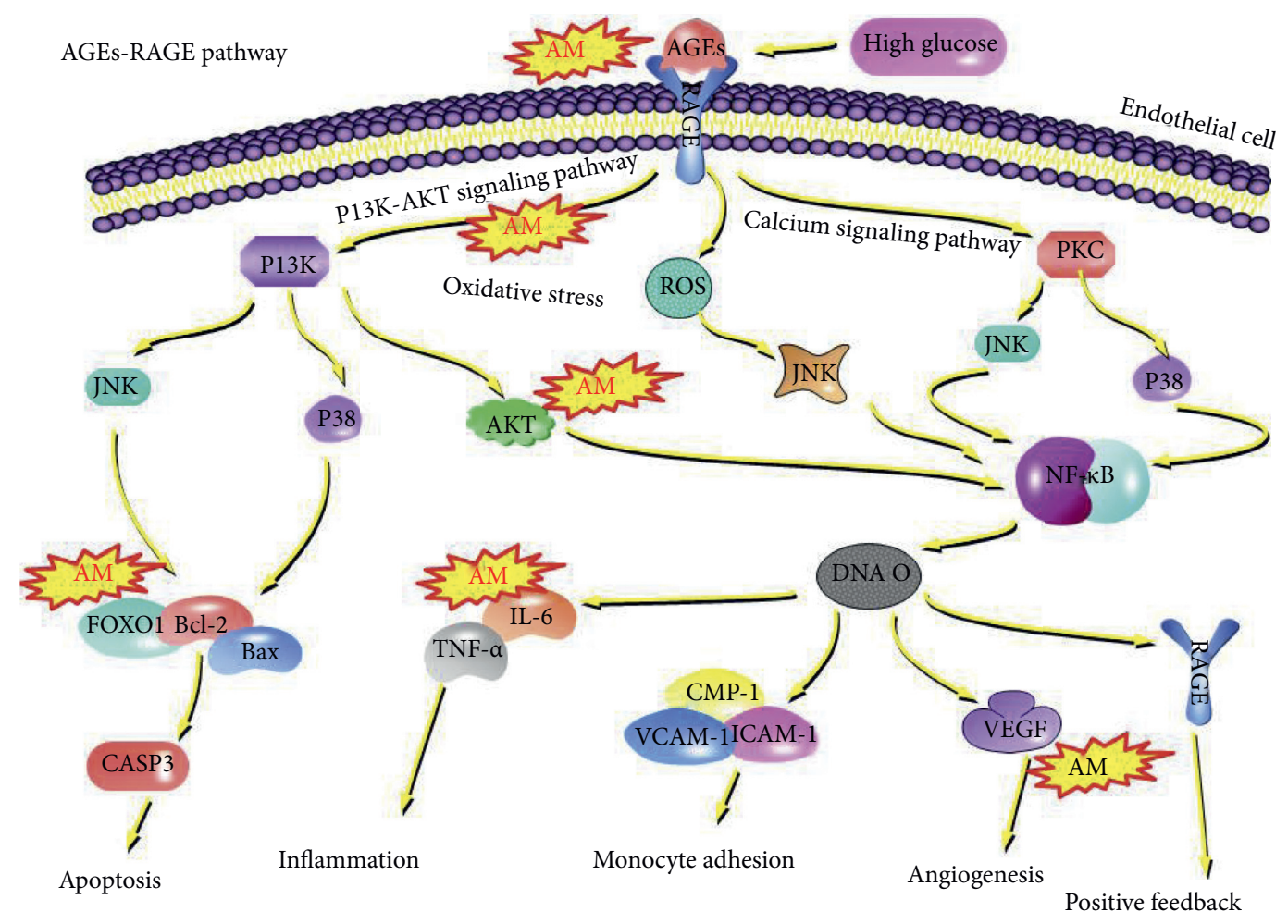

Figure 8: AGEs-RAGE pathway. Cited from the KEGG database (https://www.kegg.jp/). The position of the yellow icon indicates that AM maybe act as a key node of the pathway.

targets and pathways of AM in DR treatment, which still need to be verified by future pharmacological studies.

\section{Conclusions}

In summary, we found that AM in DR treatment has the characteristics of multiple components, multiple targets, and multiple pathways through network pharmacology. AGE-RAGE signaling pathway in diabetic complications and PI3K-Akt signaling pathway might play an important role in the treatment process, providing a theoretical basis for AM in DR treatment and a new direction for the development of drugs for DR.

\section{Data Availability}

The data of this study are available from the corresponding author upon reasonable request.

\section{Conflicts of Interest}

The authors declare that there are no conflicts of interest regarding the publication of this paper.

\section{Authors' Contributions}

QJ, XFH, and LKX conceived and designed the study; QJ and JX wrote the paper; MS and HY organized the data, SHW, GPW, and MLM analyzed the data. All authors had read and approved the final manuscript.

\section{Acknowledgments}

This study was supported by the National Natural Science Foundation of China (No. 81603666) and Capital Clinical Applied Research (No. Z181100001718183).

\section{References}

[1] GBD 2016 Disease and Injury Incidence and Prevalence Collaborators, "Global, regional, and national incidence, prevalence, and years lived with disability for 328 diseases and injuries for 195 countries, 1990-2016: a systematic analysis for the Global Burden of Disease Study 2016," Lancet, vol. 390, no. 10100, pp. 1211-1259, 2017. 
[2] Y. Li, D. Teng, X. Shi et al., "Prevalence of diabetes recorded in mainland China using 2018 diagnostic criteria from the American Diabetes Association: national cross sectional study," British Medical Journal, vol. 369, p. 997, 2020.

[3] J. Cui, J. P. Ren, D. N. Chen et al., "Prevalence and associated factors of diabetic retinopathy in Beijing, China: a crosssectional study," British Medical Journal Open, vol. 7, no. 8, p. e015473, 2017.

[4] N. Cheung, P. Mitchell, and T. Y. Wong, "Diabetic retinopathy," The Lancet, vol. 376, no. 9735, pp. 124-136, 2010.

[5] S. Vujosevic, S. J. Aldington, P. Silva et al., "Screening for diabetic retinopathy: new perspectives and challenges," The Lancet Diabetes \& Endocrinology, vol. 8, no. 4, pp. 337-347, 2020.

[6] W. Wang and A. Lo, "Diabetic retinopathy: pathophysiology and treatments," International Journal of Molecular Sciences, vol. 19, no. 6, p. 1816, 2018.

[7] American Diabetes Association, "Classification and diagnosis of diabetes: standards of medical care in diabetes-2019," Diabetes Care, vol. 42, no. Suppl 1, pp. S13-S28, 2019.

[8] E. Xiao and L. Luo, "Alternative therapies for diabetes: a comparison of western and traditional Chinese medicine (TCM) approaches," Current Diabetes Reviews, vol. 14, no. 6, pp. 487-496, 2018.

[9] B. Pang, Q. W. Li, Y. L. Qin et al., "Traditional Chinese medicine for diabetic retinopathy: a systematic review and meta-analysis," Medicine (Baltimore), vol. 99, no. 7, p. e19102, 2020.

[10] H. W. Zhang, H. Zhang, S. J. Grant, X Wan, and G Li, "Single herbal medicine for diabetic retinopathy," The Cochrane Database of Systematic Reviews, vol. 12, no. 12, p. CD007939, 2018.

[11] Y. Liang, Q. Zhang, L. Zhang et al., "Astragalus membranaceus treatment protects Raw264.7 cells from influenza virus by regulating G1 phase and the TLR3-mediated signaling pathway," Evid Based Complement Alternative Medicine, vol. 2019, Article ID 2971604, 10 pages, 2019.

[12] J. Li, Y. Huang, S. Zhao et al., "Based on network pharmacology to explore the molecular mechanisms of astragalus membranaceus for treating T2 diabetes mellitus," Annals of Translational Medicine, vol. 7, no. 22, p. 633, 2019.

[13] T. Behl and A. Kotwani, "Chinese herbal drugs for the treatment of diabetic retinopathy," Journal of Pharmacy and Pharmacology, vol. 69, no. 3, pp. 223-235, 2017 Mar.

[14] T. Behl and A. Kotwani, "Chinese herbal drugs for the treatment of diabetic retinopathy," Journal of Pharmacy and Pharmacology, vol. 69, no. 3, pp. 223-235, 2017.

[15] Z. Zhou, B. Chen, S. Chen et al., "Applications of network pharmacology in traditional Chinese medicine research," Evidence-Based Complementary and Alternative Medicine, vol. 2020, Article ID 1646905, 7 pages, 2020.

[16] D. Gfeller, O. Michielin, and V. Zoete, "Shaping the interaction landscape of bioactive molecules," Bioinformatics, vol. 29, no. 23, p. 3073, 2013.

[17] J. Ru, P. Li, J. Wang et al., "TCMSP: a database of systems pharmacology for drug discovery from herbal medicines," Journal Cheminform, vol. 6, p. 13, 2014.

[18] X. Xu, W. Zhang, C. Huang et al., "A novel chemometric method for the prediction of human oral bioavailability," International Journal of Molecular Sciences, vol. 13, no. 6, pp. 6964-6982, 2012.

[19] Y. Yamanishi, M. Kotera, M. Kanehisa et al., "Drug-target interaction prediction from chemical, genomic and pharmacological data in an integrated framework," Bioinformatics, vol. 26, no. 12, pp. i246-i254, 2010.

[20] S. Kim, J. Chen, T. Cheng et al., "PubChem 2019 update: improved access to chemical data," Nucleic Acids Research, vol. 47, no. D1, pp. D1102-D1109, 2019.

[21] A. Daina, O. Michielin, and V. Zoete, "SwissTargetPrediction: updated data and new features for efficient prediction of protein targets of small molecules," Nucleic Acids Research, vol. 47, no. W1, pp. W357-W364, 2019.

[22] G. Stelzer, N. Rosen, I. Plaschkes et al., "The GeneCards suite: from gene data mining to disease genome sequence analyses," Current Protocols in Bioinformatics, vol. 54, pp. 1-30, 2016.

[23] J. S. Amberger, C. A. Bocchini, F. Schiettecatte, A. F. Scott, and A. Hamosh, "OMIM.org: online Mendelian Inheritance in Man (OMIM), an online catalog of human genes and genetic disorders," Nucleic Acids Research, vol. 43, no. D1, p. D789, 2015.

[24] P. Bardou, J. Mariette, F. Escudié, C. Djemiel, and C. Klopp, "Jvenn: an interactive Venn diagram viewer," BioMed Central Bioinformatics, vol. 15, no. 1, p. 293, 2014.

[25] C. von Mering, L. J. Jensen, B. Snel et al., "STRING: known and predicted protein-protein associations, integrated and transferred across organisms," Nucleic Acids Research, vol. 33, pp. D433-D437, 2005.

[26] M. Legeay, N. T. Doncheva, J. H. Morris, and L. J. Jensen, "Visualize omics data on networks with omics visualizer, a Cytoscape app," F1000Research, vol. 9, p. 157, 2020.

[27] Y. Zhou, B. Zhou, L. Pache et al., "Metascape provides a biologist-oriented resource for the analysis of systems-level datasets," Nature Communications, vol. 10, no. 1, p. 1523, 2019.

[28] L. Zhang, D. Lu, M. Liu, M. Zhang, and Q. Peng, "Identification and interaction analysis of key miRNAs in medullary thyroid carcinoma by bioinformatics analysis," Molecular Medicine Reports, vol. 20, no. 3, pp. 2316-2324, 2019.

[29] E. Wang, L. Wang, R. Ding et al., "Astragaloside IV acts through multi-scale mechanisms to effectively reduce diabetic nephropathy," Pharmacological Research, vol. 157, p. 104831, 2020.

[30] W. Wang and A. Lo, "Diabetic retinopathy: pathophysiology and treatments," International Journal of Molecular Sciences, vol. 19, no. 6, p. 1816, 2018.

[31] B. Chen, T. He, Y. Xing, and T Cao, "Effects of quercetin on the expression of MCP-1, MMP-9 and VEGF in rats with diabetic retinopathy," Experimental and Therapeutic Medicine, vol. 14, no. 6, pp. 6022-6026, 2017.

[32] G. Lupo, M. T. Cambria, M. Olivieri et al., "Anti-angiogenic effect of quercetin and its 8-methyl pentamethyl ether derivative in human microvascular endothelial cells," Journal of Cellular and Molecular Medicine, vol. 23, no. 10, pp. 65656577, 2019.

[33] X. Wang, H. Li, H. Wang, and J. Shi, "Quercetin attenuates high glucose-induced injury in human retinal pigment epithelial cell line ARPE-19 by up-regulation of miR-29b," The Journal of Biochemistry, vol. 167, no. 5, pp. 495-502, 2020.

[34] W. Du, Y. An, X. He et al., "Protection of kaempferol on oxidative stress-induced retinal pigment epithelial cell damage," Oxidative Medicine and Cellular Longevity, vol. 2018, Article ID 1610751, 14 pages, 2018.

[35] X. H. Xu, C. Zhao, Q. Peng et al., "Kaempferol inhibited VEGF and PGF expression and in vitro angiogenesis of HRECs under diabetic-like environment," Brazilian Journal of Medical and Biological Research, vol. 50, no. 3, p. e5396, 2017. 
[36] Y. Ding, S. Yuan, X. Liu et al., "Protective effects of astragaloside IV on $\mathrm{db} / \mathrm{db}$ mice with diabetic retinopathy," PLoS One, vol. 9, no. 11, p. e112207, 2014.

[37] Y. Qiao, C.-L. Fan, and M.-K. Tang, "Astragaloside IV protects rat retinal capillary endothelial cells against high glucoseinduced oxidative injury," Drug Design, Development and Therapy, vol. 11, pp. 3567-3577, 2017.

[38] N. Han, H. Xu, N. Yu, Y. Wu, and L. Yu, "MiR-203a-3p inhibits retinal angiogenesis and alleviates proliferative diabetic retinopathy in oxygen-induced retinopathy (OIR) rat model via targeting VEGFA and HIF-1 $\alpha$," Clinical and Experimental Pharmacology and Physiology, vol. 47, no. 1, pp. 85-94, 2020.

[39] W. Xie, P. Zhou, M. Qu et al., "Ginsenoside Re attenuates high glucose-induced RF/6A injury via regulating PI3K/AKT inhibited HIF-1 $\alpha$ /VEGF signaling pathway," Front Pharmacology, vol. 11, p. 695, 2020.

[40] M. Tian, S. Liu, L Liu et al., "Correlations of the severity of diabetic retinopathy with EPO, Caspase-3 expression and oxidative stress," European Review for Medical and Pharmacological Sciences, vol. 23, no. 22, pp. 9707-9713, 2019.

[41] Y. Wang, W. L. Zhai, and Y. W. Yang, "Association between NDRG2/IL-6/STAT3 signaling pathway and diabetic retinopathy in rats," European Review for Medical and Pharmacological Sciences, vol. 24, no. 7, pp. 3476-3484, 2020.

[42] O. M. Cecilia, C. G. José Alberto, N. P. José et al., "Oxidative stress as the main target in diabetic retinopathy pathophysiology," Journal of Diabetes Research, vol. 2019, Article ID 8562408, 21 pages, 2019.

[43] Z. Li, Y. Li, J. M. Overstreet et al., "Inhibition of epidermal growth factor receptor activation is associated with improved diabetic nephropathy and insulin resistance in type 2 diabetes," Diabetes, vol. 67, no. 9, pp. 1847-1857, 2018.

[44] G. Giurdanella, F. Lazzara, N. Caporarello et al., "Sulodexide prevents activation of the PLA2/COX-2/VEGF inflammatory pathway in human retinal endothelial cells by blocking the effect of AGE/RAGE," Biochemical Pharmacology, vol. 142, pp. 145-154, 2017. 